\title{
TUTORIAL
}

\section{Corticofugal system and processing of behaviorally relevant sounds : Perspective}

\author{
Nobuo Suga, Enquan Gao ${ }^{1}$, Xiaofeng Ma, Masashi Sakai and Syed A. Chowdhury ${ }^{2}$ \\ Department of Biology, Washington University, \\ One Brookings Drive, St. Louis, MO 63130 USA \\ e-mail: suga@biology.wustl.edu \\ ${ }^{1}$ Present address: Department of Neurology, General Hospital of the Navy, Beijing 100037, \\ P. R. China \\ ${ }^{2}$ Present address: Department of Physiology and Biophysics, Dalhousie University, Halifax, \\ NS, Canada B3H $4 H 7$
}

\begin{abstract}
The auditory system consists of the ascending and descending (corticofugal) systems. One of the major functions of the corticofugal system is the adjustment and improvement of auditory signal processing in the subcortical auditory nuclei, i.e., the adjustment and improvement of the input of cortical neurons. The corticofugal system evokes a small, short-term reorganization (plasticity) of the inferior colliculus, medial geniculate body and auditory cortex for acoustic signals repetitively delivered to an animal. When these signals become behaviorally relevant to the animal through conditioning (associative learning), the short-term reorganization is augmented and changes into a long-term reorganization of the auditory cortex. Animals acquire the behavioral relevance of sounds through associative learning. Human babies also acquire language through associative learning. Therefore, the corticofugal system is expected to play a particularly important role in processing behaviorally relevant sounds and in reorganizing the auditory cortex according to the behavioral relevance of sounds. Since the ascending and descending systems form multiple feedback loops, the neural mechanisms for auditory information processing cannot be adequately understood without the exploration of the interaction between the ascending and descending systems.
\end{abstract}

Keywords: Auditory system, Communication sound, Hearing, Plasticity, Speech

PACS number: 43.64.Qh, 43.71.Pc

\section{INTRODUCTION}

The auditory system consists of the ascending and descending (corticofugal) systems. The corticofugal system, originating from the auditory cortex (AC), forms multiple feedback loops to modulate auditory signal processing at the medial geniculate body (MGB), inferior colliculus (IC), superior olivary complex (SOC), dorsal cochlear nucleus (DCN) and cochlea. Since the functional role of the corticofugal system has not yet been well studied, the extent to which auditory signals are processed by the ascending system alone is not clear. The first aim of this article is to enumerate problems and recent progress in the research of corticofugal modulation, not to review the data, because a review article on corticofugal modulation has recently been written (Suga et al., 2000). (Please note that only review articles are cited in this article because of the page limitations of this journal.)

One of the possible functions of the corticofugal sys- tem is the adjustment of the functional organization of the $\mathrm{AC}$ according to auditory experience. Language acquisition is based on an enormous amount of associative learning of acoustic patterns. Associative learning changes the organization of the AC via the corticofugal auditory system and other systems (Suga et al., 2000). Considering such changes (plasticity) and neuroethological data related to neural mechanisms for processing behaviorally relevant sounds, the simple spatiotemporal pattern hypothesis based on the "pure-tone" data obtained from the primary auditory cortex of the cat and other mammalian species may be misleading for speech scientists and may contribute little to the understanding of the processing of behaviorally relevant sounds. The second aim of this article is to express our opinion related to the neural mechanisms for the processing of behaviorally relevant sounds, including speech sounds. 


\section{NEURONS TUNED TO PARAMETERS OR ELEMENTS CHARACTERIZING BEHAVIORALLY RELEVANT SOUNDS}

All peripheral neurons are tuned to single frequencies (best frequencies: BF's). In the central auditory system, excitatory, inhibitory and facilitatory neural interactions take place at multiple levels and produce neurons with sharp "level-tolerant" frequency tuning and also neurons tuned to specific values of parameters other than frequency. Some of these are apparently tuned to the information-bearing parameters (IBP's) or elements (IBE's) characterizing behaviorally relevant sounds, as found in mammals (Suga, 1988, 1994; Covey and Casseday, 1999; Wang, 2000; Rauschecker and Tian, 2000), avians (Solis et al., 2000), amphibians and insects.

In a cricket, a repetition rate or rhythm of stridulatory sounds is an IBP for detecting a species-specific call. The peripheral neurons of the cricket act as an "allpass" filter responding to repetition rates of sounds in a wide range, but its central nervous system has neurons acting as a band-pass filter which is tuned to a repetition rate of the species-specific stridulatory sound. The frog's central auditory system has neurons tuned to a repetition rate of species-specific calls or to a combination of the components of species-specific calls. "Combinationsensitive" neurons are defined as neurons showing a facilitative response to a combination of more than one sound. (Facilitative response is defined as the response to a combination of sounds that is larger than the algebraic sum of responses to individual sounds to be combined.) Combination-sensitive neurons have been found not only in frogs, but also in zebra finches (Solis et al., 2000), mustached bats (Suga, 1988, 1994), big brown bats, marmosets (Wang, 2000) and macaque monkeys (Rauschecker and Tian, 2000). Many species of mammals, including humans, produce complex sounds for communication. Frequency-modulated (FM) calls make a call repertoire rich. Microchiropterans emit short FM sounds for echolocation. In their brain, neurons tuned to specific FM sounds (e.g., an upward- or downwardsweeping FM sound) have been found (Suga, 1973). In primates, it has been known that upward-sweeping and downward-sweeping FM sounds convey totally different information to them. In the $\mathrm{AC}$ of marmosets (Wang, 2000) and macaque monkeys (Rauschecker and Tian, 2000), FM-specialized (or -sensitive) neurons were also found. In zebra finches, combination-sensitive neurons responding best to an animal's own song are produced in the lateral magnocellular nucleus of the anterior neostriatum according to song learning (Solis et al., 2000). All the neurophysiological findings enumerated above were made only in the experiments with acoustic stimuli simulating species-specific calls, and strongly suggest that combination-sensitive neurons and FM-specialized neurons play an important role in processing complex sounds and the temporal pattern of sounds. This may also be true for processing speech sounds in the human brain (Suga, 1988).

\section{MULTIPLE COCHLEOTOPIC (FREQUENCY) AND COMPUTATIONAL MAPS AND SPATIOTEMPORAL PATTERN OF NEURAL ACTIVITY}

The mammalian AC consists of multiple areas. Therefore, it has been speculated that these areas differ from each other in processing auditory information. The functional organization of the AC beyond a cochleotopic (frequency or tonotopic) map has been explored in different species of animals to some extent. Among them, the $\mathrm{AC}$ of the mustached bat (Pteronotus parnellii) has perhaps been best explored in terms of both multiple cochleotopic and computational maps.

In the mustached bat, the AC shows the following important facts in organization. (i) The frequency axes in the $\mathrm{AC}$ are not exact copies of the frequency axis along the basilar membrane. Certain portions of it are shrunken or stretched. (ii) Different portions of the peripheral frequency axis are superimposed in parallel or orthogonally across certain cortical areas. (iii) The ratios of two frequencies are expressed by diagonal spatial patterns of activated neurons in frequency-vs.-frequency coordinates. (iv) An area where the frequency axis can hardly be demonstrated with single tone stimuli has frequency axes when detailed studies are performed with paired stimuli. (v) The complex multiple frequency representations in the AC are directly related to the representations of different types of auditory information. (vi) Different types of neurons tuned to IBP's are clustered in different areas of the AC and form IBP (computational) maps (Suga, 1988, 1994).

The AC apparently shares the basic neural mechanisms and organizational principles with the visual cortex. For example, the AC of the mustached bat has different areas specialized for processing different features of biosonar images such as distance and velocity (Suga, 1988, 1994), whereas the visual cortex of monkeys has different areas specialized for processing different features of visual images such as motion, form and color (Livingstone and Hubel, 1988).

As described above, the central auditory system produces various types of neurons that are tuned to a particular value of IBP or particular IBE, and that are located in particular subdivisions or areas of the central au- 
ditory system. They are most strongly excited by speciesspecific sounds. However, their filter properties are not sharp, so that they are also excited by other sounds. Therefore, an IBP or IBE is appropriately expressed by the spatiotemporal pattern of activities of IBP- or IBEtuned neurons.

In the primary $\mathrm{AC}$ of cats and other mammalian species, responses of single neurons and spatiotemporal patterns of neural activity have been studied for tone bursts. There is no question about the importance of spatiotemporal pattern of neural activity in representing (processing) acoustic stimuli. However, such studies have not been designed to explore the neural mechanisms for processing behaviorally relevant sounds and may mislead non-neurophysiologists to conclude that simple spatiotemporal pattern of neural activity in the primary AC itself is sufficient and is directly related to the processing of behaviorally relevant sounds.

The peripheral auditory system only has an anatomical axis for frequency and an array of neurons tuned in frequency. The activity of individual peripheral neurons cannot uniquely express the properties of an acoustic signal. For instance, a peripheral neuron tuned to a $5 \mathrm{kHz}$ sound responds not only to a pure tone of $5 \mathrm{kHz}$, but also to an FM sound sweeping across $5 \mathrm{kHz}$ regardless of sweep direction and to a noise burst containing a $5 \mathrm{kHz}$ component regardless of bandwidth. Therefore, the neuron cannot code the type of acoustic signal stimulating the ear. A pure tone of $5 \mathrm{kHz}$, for instance, is expressed only by both the excitation of neurons tuned to $5 \mathrm{kHz}$ and non-excitation of neurons tuned to other frequencies. The properties of an acoustic signal are appropriately represented (coded) only by the spatiotemporal pattern of activity of all peripheral neurons. Most neurons in the primary AC respond to many different types of sounds, regardless of their behavioral relevance. The properties of an acoustic signal are also appropriately represented only by the spatiotemporal pattern of neural activity in the primary AC. However, it is unsatisfactory to conclude that these sounds are expressed or processed by spatiotemporal pattern of neural activity in the primary AC, ignoring the presence of IBP- or IBE-tuned neurons. Research exploring the response properties of individual neurons contributing to the spatiotemporal pattern of neural activity in non-primary auditory cortices is long overdue.

As described already in this article, the central auditory system produces neurons with response properties which are quite different from those of peripheral neurons. In the $\mathrm{AC}$ of the mustached bat, for instance, IBPor IBE-tuned neurons contributing to the spatiotemporal pattern of neural activity representing behaviorally relevant sounds are quite different in response properties from peripheral neurons and are clustered in different areas of the AC (Suga, 1988, 1994). Since IBP- or IBE-tuned neurons are poorly excited by behaviorally irrelevant sounds, but best excited by behaviorally relevant sounds, they can make an animal more strongly attend to behaviorally relevant sounds than to irrelevant sounds. Since different types of IBP- or IBE-tuned neurons are clustered in separate areas or subdivisions in the central auditory system, their tuning to a particular IBP or IBE can be sharpened by lateral inhibition. These are the main advantages in representing auditory information by the spatiotemporal pattern of activity of IBP- or IBE-tuned neurons.

\section{MULTIPLE FEEDBACK LOOPS FORMED BY THE CORTICOFUGAL AUDITORY SYSTEM}

The response properties of neurons and the computational maps in the central auditory system have been explained to be produced by mechanisms based on divergent and convergent projections of neurons in the ascending auditory system for inhibition, coincidence detection (facilitation), delay lines, and disinhibition (Suga, 1973, 1994). Since the basic response properties of cortical auditory neurons have been found to be mostly produced in the subcortical auditory nuclei (Covey and Casseday, 1999), they are expected to be under corticofugal modulation. As a matter of fact, recent findings indicate that the corticofugal system plays important roles in shaping (or even producing) the response properties of central auditory neurons and in reorganizing the cochleotopic and computational maps (Suga et al., 1997, 2000).

Neurons in the deep layers of the AC project to the medial geniculate body (MGB), inferior colliculus (IC) or subcollicular auditory nuclei. These corticofugal projections are cochleotopically (tonotopically) organized. Corticothalamic fibers project only to the ipsilateral MGB and thalamic reticular nucleus. However, corticocollicular fibers bilaterally project to the IC. The ipsilateral projection is much more extensive and topographically organized than the contralateral projection. Therefore, ipsilateral corticofugal modulation is expected to be much larger than contralateral corticofugal modulation in the IC and MGB, and to be frequency-dependent. Corticofugal projections are bilateral to the subcollicular nuclei: superior olivary complex and cochlear nucleus. Corticofugal modulation is expected to take place even in the cochlea via olivocochlear neurons in the superior olivary complex. The central nucleus of the IC projects not only to the MGB and the superior colliculus, but also to medial olivocochlear neurons, which mostly project to contralateral cochlear outer hair cells. In general, olivocochlear neurons bilaterally project to the cochlea, although there 
are some differences in olivocochlear projections between species (Huffman and Henson, 1990; Warr, 1992).

To study the functional roles of the corticofugal system, one should not ignore that corticofugal and subcortical neurons are both tuned to particular values of an acoustic parameter. Therefore, electrical stimulation or drug application for activation or inactivation should be highly focal, except for the initial phase of neurophysiological research on the corticofugal system, and corticofugal effects on subcortical neurons should be evaluated with regard to the relationship in tuning between stimulated or inactivated cortical neurons and recorded subcortical neurons. The recent research designed on this philosophy resulted in several findings in the mustached bat and the big brown bat, Eptesicus fuscus (Suga, et al. 2000). However, the exploration of corticofugal functions is not yet satisfactory. An obvious critical experiment to be performed is the selective inactivation of individual feedback loops without injuring the ascending auditory system. Such an experiment, however, has not yet been performed because of anatomical complexity.

\section{CORTICOFUGAL FUNCTIONS WHICH HAVE BEEN CONSIDERED}

The corticofugal function for hearing remains to be further studied in terms of the following subfunctions.

a. Egocentric selection for "short-term" adjustment and improvement of auditory signal processing according to auditory experience. Egocentric selection is performed by the AC and the corticofugal system. It is based on focused positive feedback associated with lateral inhibition (Suga et al., 1997). Egocentric selection adjusts and improves cortical neurons' own input, so that it is similar to the function of corticofugal feedback in the visual system.

b. Reorganization for "long-term" adjustment and improvement of signal processing. Long-term reorganization (plasticity) of the $\mathrm{AC}$ is based on egocentric selection working together with non-auditory systems, i.e., on activities of the corticofugal system, cholinergic basal forebrain and others (Suga et al., 2000).

c. Gain control or gating. Overall facilitative or inhibitory corticofugal modulation indicate this function. Egocentric selection can be viewed as selective gain control. The corticofugal visual system also performs gain control.

d. Shaping (or even producing) response properties of neurons, in particular, of combination-sensitive neurons. Filter properties of neurons in the frequency, amplitude, time and spatial domains can be sharpened by the corticofugal system. Sharpening of tuning is one of the functions of egocentric selection. The production of combination-sensitivity through interactions between the ascending and descending systems must be a particularly important function for processing behaviorally relevant complex sounds (Suga et al., 1997, 2000). In the visual system, response properties of thalamic neurons become complex because of corticofugal feedback.

e. Binding of the different features of auditory signals. The problem of binding has been extensively studied in the visual system, but not in the auditory system. In the visual system, the corticofugal system evokes featurelinked synchronized discharges in the thalamic neurons.

f. Stabilization of thalamic auditory responses via the thalamic reticular nucleus. The thalamic reticular nucleus receives axon collaterals from both ascending thalamo-cortical fibers and descending cortico-thalamic fibers. Corticofugal positive feedback has a high gain, so that ringing would be evoked if it is not incorporated with inhibition through the thalamic reticular nucleus. If the thalamic reticular nucleus does not operate properly, long-lasting discharges, perhaps responsible for tinnitus, would be produced. Cooling of the AC had complex effects on the auditory responses of neurons in the MGB and the thalamic reticular nucleus, so that the reticular nucleus is hypothesized to act as an adaptive filter.

g. Attentional modulation of auditory signal processing. In cats, visual attention to a mouse reduces auditory responses of the dorsal cochlear nucleus, and a visual discrimination task reduces auditory nerve responses to clicks. In humans, visual attention reduces auditory nerve responses and otoacoustic emissions evoked by clicks. The corticofugal system probably mediates attentional modulation of auditory signal processing.

h. Low frequency modulation of brain rhythm. The corticofugal system transmits slow oscillatory changes in cortical activity to the thalamic visual nucleus. This slow oscillation $(0.6-1.0 \mathrm{~Hz})$ interacts with spindles $(7-14 \mathrm{~Hz})$ generated in the thalamus, modulates neural excitability, and produces different brain rhythms characterizing various behavioral states (Steriade, 1999).

During the last three years, we have been studying egocentric selection and the reorganization (plasticity) of the AC by the corticofugal system. The corticofugal system appears to play an essential role in adjusting and improving the processing of behaviorally relevant sounds according to auditory experience. Therefore, in the following we will first summarize our working hypothesis and will write our view of the contribution of comparative auditory neuroethology to the understanding of speechsound processing. 


\section{THE CORTICOFUGAL SYSTEM AND PLASTICITY}

Gao and Suga proposed the following working hypothesis of the corticofugal function for cortical plasticity. When behaviorally irrelevant acoustic stimuli are delivered to an animal, auditory signals representing the stimuli ascend from the cochlea to the AC. Then, the $\mathrm{AC}$ and the corticofugal system perform egocentric selection, which is a small and short-term modulation of subcortical signal processing. Accordingly, the small and short-term cortical change is evoked. When the acoustic stimuli are paired with electric leg-stimulation, the auditory and somatosensory signals ascend from the periphery to the auditory and somatosensory cortices, respectively, and then to the amygdala through association cortices. These signals are perhaps associated in the association cortices and the amygdala which is essential for evoking a conditioned behavioral response. Therefore, the acoustic stimuli become behaviorally relevant to the animal (Suga et al., 2000). The amygdala sends the "associated" signal to the cholinergic basal forebrain, which increases the cortical acetylcholine level. Then, the change in the AC is augmented (Weinberger, 1995, 1998). Accordingly, egocentric selection is augmented, and the subcortical change becomes larger, so that the cortical change becomes larger and long-term. This positive feedback loop is controlled by inhibition mediated by the thalamic reticular nucleus. What is particularly important is that the cortical change becomes large and long-lasting for an acoustic signal when it becomes behaviorally relevant to an animal. As already described in the present article, the corticofugal system plays an important role in shaping (even producing) response properties of central auditory neurons (Suga et al., 1997). Therefore, it may be predicted that particular response properties of central auditory neurons are produced during the development of the auditory system through corticofugal modulation based on auditory experience.

Pathways through the multi-sensory thalamic nuclei, which have been considered to be essential for cortical plasticity by Weinberger $(1995,1998)$, appeared to be quite reasonable to us. However, the importance of these pathways is doubtful because of the following data obtained from the big brown bat. (i) Inactivation or activation of the somatosensory cortex respectively abolishes or augments cortical and collicular BF shifts caused by fear conditioning. Therefore, the somatosensory cortex as well as the AC plays an essential role in cortical plasticity caused by the conditioning. (ii) Collicular changes evoked by the corticofugal system precede cortical changes. (iii) The AC and corticofugal system have an intrinsic mechanism for cortical and subcortical plastic changes which are highly specific to the parameters characterizing an acoustic stimulus. (iv) Fear conditioning evokes plasticity of the IC which is the nucleus one step below the thalamus. In other words, the multi-sensory thalamic nuclei are not the first place where the plasticity due to the conditioning is evoked (Suga et al., 2000).

\section{DIFFERENCES IN CORTICOFUGAL MODULATION BETWEEN SPECIES AND BETWEEN ORDINARY AND SPECIALIZED AREAS OF A SINGLE SPECIES}

Egocentric selection has been found not only in the mustached bat and big brown bat, but also in the cat, so that it may be a general function of the corticofugal system. Corticofugal positive feedback associated with lateral inhibition has also been found in the visual system. However, the effect of egocentric selection on the cochleotopic (frequency) map is different between different species of mammals and between non-specialized (ordinary) and specialized areas of the AC of a single species (Suga et al., 2000). In the big brown bat and the Mongolian gerbil, the reorganization of the cochleotopic map evoked by focal electrical stimulation of the $\mathrm{AC}$ is centripetal in the IC and AC. [A shift in best frequency (BF) of a neuron toward the $\mathrm{BF}$ of electrically stimulated cortical neurons is called a "centripetal BF shift", whereas a shift in BF away from that is called a "centrifugal BF shift."] In the mustached bat, the reorganization of the cochleotopic map evoked by focal electric stimulation of the $\mathrm{AC}$ is also centripetal in the posterior division of the primary $\mathrm{AC}$ which is similar to the $\mathrm{AC}$ of many other mammalian species in frequency representation, whereas it is centrifugal in the DSCF area of the primary AC which over-represents sound at $\sim 61 \mathrm{kHz}$. The FM-FM area of the AC of the mustached bat has an echo-delay map instead of a frequency map. The reorganization of the echo-delay map evoked by focal electric stimulation of the FM-FM area is centrifugal. The DSCF and FM-FM areas are both specialized for the representation of specific biosonar information and are large relative to the rest of the AC (Suga, 1988, 1994). Therefore, our findings indicate that cortical reorganization according to auditory experience can be quite different between specialized and non-specialized (i.e., ordinary) cortical auditory areas. 


\section{CONTRIBUTION OF COMPARATIVE AUDITORY NEUROETHOLOGY TO THE UNDERSTANDING OF SPEECH-SOUND PROCESSING}

The reorganization of the central auditory system is augmented by behavioral relevance (associative learning) of acoustic signals (Weinberger, 1995; Suga et al., 2000), and the reorganization differs between species and between cortical areas of a single species (Suga et al., 2000). These two findings suggest that the functional organization of the $\mathrm{AC}$ of an adult animal is more related to the processing of behaviorally relevant sounds such as communication sounds and orientation sounds; that it can be different between species according to differences in behaviorally relevant sounds between them; that organization and reorganization of the cortical areas specialized for the processing of behaviorally relevant sounds (e.g., speech) is quite different from those of the primary auditory cortex; and that the $\mathrm{AC}$ of an awake animal should be studied with acoustic stimuli appropriately designed simulating behaviorally relevant sounds.

Human infants learn language by hearing words (nonsense syllables for the infants without associative learning) while seeing, touching, tasting and/or smelling objects. Language acquisition is based on an enormous amount of associative learning. Therefore, response properties of cortical neurons and functional organization of the AC of humans may result from corticofugal modulation of speech processing which is augmented by associative learning. Progress in functional magnetic resonance imaging, fMRI, has been remarkable. Therefore, one may directly work on humans to understand the neural mechanisms for processing speech in humans. However, the understanding of the neural mechanisms producing particular types of response properties of cortical neurons may have to be based upon animals data.

The primary aim of auditory neuroethology is to explore the neural mechanisms for the auditory behavior of a given species of animal, not to obtain data valuable to understanding speech processing. However, neuroethological studies on the auditory systems of different types of animals, including monkeys, will be successful in identifying which neural mechanisms are shared by different types of animals and which mechanisms are speciesspecific. Therefore, comparative auditory neuroethology may yield a valuable insight into possible neural mechanisms of speech-sound processing.

Speech scientists appear to claim that the neural mechanisms for hearing found in non-human primates are relevant to processing speech, but those found in nonprimates are not. However, IBP- or IBE-tuned neurons first found in non-primate animals (Suga, 1988) have also recently been found in non-human primates (Wang, 2000; Rauschecker and Tian, 2000). Therefore, the neural mechanisms for processing speech have presumably developed on top of the neural mechanisms for processing behaviorally relevant sounds in animals, including nonprimate animals.

Hemispheric asymmetry had been considered to exist only in the human brain, and to make the human brain unique. However, hemispheric asymmetry has been found not only in the macaque monkey, but also in the house mouse and the Mongolian gerbil. It is expected to be found in many mammalian species, if auditory physiology is appropriately performed with behaviorally relevant acoustic stimuli.

Human speech sounds share identical IBE's and IBP's with animal sounds. However, human speech is unique because in speech, many different types of phonemes are sequenced in enormous variations, and there are, accordingly, a huge number of combinations of FM components. On the contrary, in animals including monkeys, the sequence of sounds is nearly fixed. Therefore, studying the monkey's AC with human speech as stimuli may contribute little to understanding the neural mechanisms for the processing of human speech in the human AC. It is much more important to study how the monkey's AC processes species-specific calls. In general, research on the AC should be focused on how behaviorally relevant sounds are processed in the AC.

In the cerebral cortex of a mammal, there are many auditory areas: at least 14 physiologically identified areas in the mustached bat (Suga, 1994) and at least 15 anatomically identified areas in the monkey, excluding areas in the frontal cortex (Kaas and Hackett, 2000). The research with behaviorally relevant acoustic stimuli should focus on non-primary auditory areas. Otherwise, important neural mechanisms for the processing of behaviorally relevant sounds appear to be hardly explored.

\section{ACKNOWLEDGEMENT}

We thank Mr. Nate Laleman for editing the manuscript. Our research on the auditory system has been supported by a research grant from the National Institute on Deafness and Other Communicative Disorders (DC 00175).

\section{REFERENCES}

Covey, E. and Casseday, J. H. (1999). "Timing in the auditory system of the bat", Annu. Rev. Physiol. 61, 457-476.

Huffman, R. F. and Henson, O. W. Jr. (1990). "The descending auditory pathway and acousticomotor systems: Connections with the inferior colliculus", Brain Res. Rev. 15, 295-323.

Kaas, J. H. and Hackett, T. A. (2000). "Subdivisions of auditory cortex and processing streams in primates", Proc. Natl. Acad. Sci. 
USA 97, 11793-11799.

Livingstone, M. and Hubel, D. (1988). "Segregation of form, color, movement, and depth: Anatomy, physiology, and perception", Science 240, 740-749.

Rauschecker, J. P. and Tian, B. (2000). "Mechanisms and streams for processing of "what" and "where" in auditory cortex", Proc. Natl. Acad. Sci. USA 97, 11800-11806.

Solis, M. M., Brainard, M. S., Hessler, N. A. and Doupe, A. J. (2000). "Song selectivity and sensory-motor signals in vocal learning and production", Proc. Natl. Acad. Sci. USA 97, 1183611842.

Steriade, M. (1999). "Coherent oscillations and short-term plasticity in corticothalamic networks", Trends Neurosci. 22, 337-345.

Suga, N. (1973). "Feature extraction in the auditory system of bats", in Basic Mechanisms in Hearing, A. R. Moller, Ed. (Academic Press, New York), pp. 675-742.

Suga, N. (1988). "Auditory neuroethology and speech processing: Complex-sound processing by combination-sensitive neurons", in Auditory Function, G. M. Edelman, W. E. Gall and W. M. Cowan, Eds. (John Wiley \& Sons, New York), Chap. 23, pp. 679-720.

Suga, N. (1994). "Processing of auditory information carried by complex species-specific sounds", in The Cognitive Neuro- sciences, M. S. Gazzaniga, Ed. (MIT Press, Cambridge, Mass.), pp. 295-318.

Suga, N., Gao, E., Zhang, Y., Ma, X. and Olsen, J. F. (2000). "The corticofugal system for hearing: Recent progress", Proc. Natl. Acad. Sci. USA 97, 11807-11814.

Suga, N., Yan, J. and Zhang, Y. (1997). "Cortical maps for hearing and egocentric selection for self-organization", Trends Cognit. Sci. 1, 13-20.

Wang, X. (2000). "On cortical coding of verbal communication sounds in primates", Proc. Natl. Acad. Sci. USA 97, 11843-11849.

Warr, W. B. (1992). "Organization of olivocochlear efferent systems in mammals", in The Mammalian Auditory Pathway: Neuroanatomy, D. B. Webster, A. N. Popper and R. R. Fay, Eds. (Springer-Verlag, New York), pp. 410-448.

Weinberger, N. M. (1995). "Retuning the brain by fear conditioning", in The Cognitive Neurosciences, M. S. Gazzanica, Ed. (MIT Press, Cambridge, Mass.), pp. 1071-1089.

Weinberger, N. M. (1998). "Physiological memory in primary auditory cortex: Characteristics and mechanisms", Neurobiol. Learn. Mem. 70, 226-251. 